\title{
Pulsed beams as field probes for precision measurement
}

\author{
J. J. Hudson, H. T. Ashworth, D. M. Kara, M. R. Tarbutt, B. E. Sauer, E. A. Hinds \\ Centre for Cold Matter, The Blackett Laboratory, \\ Imperial College London, London $S W^{r}$ 2AZ, UK
}

\begin{abstract}
We describe a technique for mapping the spatial variation of static electric, static magnetic, and rf magnetic fields using a pulsed atomic or molecular beam. The method is demonstrated using a beam designed to measure the electric dipole moment of the electron. We present maps of the interaction region, showing sensitivity to (i) electric field variation of $1.5 \mathrm{~V} / \mathrm{cm}$ at $3.3 \mathrm{kV} / \mathrm{cm}$ with a spatial resolution of $15 \mathrm{~mm}$; (ii) magnetic field variation of $5 \mathrm{nT}$ with $25 \mathrm{~mm}$ resolution; (iii) radio-frequency magnetic field amplitude with $15 \mathrm{~mm}$ resolution. This new diagnostic technique is very powerful in the context of high-precision atomic and molecular physics experiments, where pulsed beams have not hitherto found widespread application.
\end{abstract}

PACS numbers: $32.60 .+\mathrm{i}, 39.10 .+\mathrm{j}$

\section{INTRODUCTION}

The structure and interactions of atoms and molecules have been studied with great precision using atomic and molecular beams 1, 2]. Some examples include accurate time-keeping [3, 4], precise tests of physical theories [5, 6], measurements of the constancy of physical constants 7-9], and searches for physics beyond the Standard Model [10-13]. These kinds of experiments require careful control and monitoring of stray and applied fields, both electric and magnetic, throughout the interaction region of the apparatus. In this paper we develop a novel method for this monitoring, based on the use of pulsed supersonic molecular beams. Such beams are well known as useful spectroscopic sources for many applications in physics and chemistry, mainly because they can provide a wide variety of molecular species at low temperature and high average intensity [1]. Here, we point out that the small spatial/temporal extent of the pulses opens a way to map electric and magnetic fields with high spatial resolution. We demonstrate this idea using a high-voltage parallel-plate structure that is also a radio-frequency ( $\mathrm{rf}$ ) transmission-line. This allows us to drive molecular hyperfine transitions anywhere in the interaction region. The line centre, lineshape and transition dynamics then provide information about the electric, magnetic and $\mathrm{rf}$ fields at the place where the transition occurs.

\section{THE BEAM MACHINE}

The apparatus used to demonstrate this method is shown schematically in Fig. 1. Its primary purpose is to measure the electric dipole moment of the electron [11], a very sensitive experiment that requires careful field mapping. A pulse of $\mathrm{YbF}$ molecules is produced in the source every $40 \mathrm{~ms}$ by laser-ablating a solid $\mathrm{Yb}$ target into a supersonically expanding gas jet of $\mathrm{Ar}$ and $\mathrm{SF}_{6}[14]$. The gas pulse passes through a $2 \mathrm{~mm}$ diameter skimmer, placed $60 \mathrm{~mm}$ from the source, in order to form a wellcollimated beam. Each pulse of YbF molecules forms in a small region over a short time (see section III) and cools to give a few times $10^{5}$ molecules at the detector in the ro-vibronic ground state. The molecular packets have a mean velocity of about $590 \mathrm{~m} / \mathrm{s}$ and a velocity spread of $40 \mathrm{~m} / \mathrm{s}$ FWHM, corresponding to a translational temperature of a few Kelvin. The length of a packet anywhere in the interaction region is therefore approximately $7 \%$ of its distance from the source.

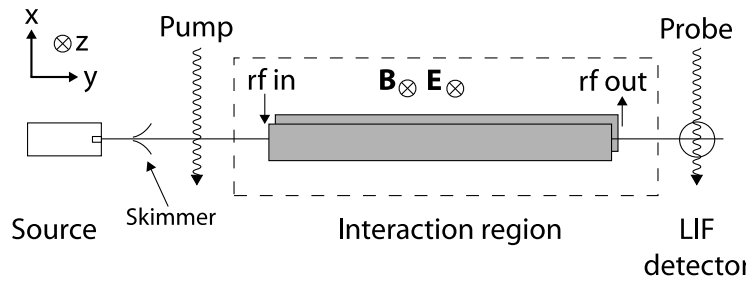

FIG. 1: Schematic diagram of the beam machine. The molecular packet issues from the source (in the y-direction) and is skimmed before being optically pumped into a single hyperfine state. The molecules enter the magnetically shielded interaction region (dashed line) and fly through a high-voltage capacitor that doubles as an rf transmission-line. The electric field points along the $\mathrm{z}$-direction. The rf-magnetic field is polarised along the $\mathrm{x}$-direction. A small $\mathrm{B}$ field can be applied in the z-direction. After leaving the interaction region the molecules are detected by laser induced fluorescence.

A single-mode continuous-wave dye laser beam, the pump shown in Fig. 1, excites the molecules downstream from the skimmer on the $A^{2} \Pi_{1 / 2}-X^{2} \Sigma^{+} \mathrm{Q}(0)$ transition at $552 \mathrm{~nm}$. The Doppler width is suppressed by pointing the pump laser beam perpendicular to the molecular beam. This gives a narrow enough linewidth to resolve the two X-state hyperfine levels $F=0$ and $F=1$, separated by $170 \mathrm{MHz}$. We tune the pump laser to excite the $F=1$ population, which becomes depleted as a result. Any remaining $F=1$ molecules are detected at the end of the $1.3 \mathrm{~m}$-long beam machine using a second laser beam of the same frequency, the probe in Fig. 1. The laser-induced fluorescence (LIF) is monitored by a photomultiplier, a typical pulse (without pump) being shown 
in Fig. 2 versus time of arrival. The $140 \mu$ s width of this pulse is essentially all due to the velocity spread of the beam. The temporal resolution of the LIF detection is $7 \mu \mathrm{s}$, and is due principally to the $4 \mathrm{~mm}$ spatial width of the probe laser beam.

LIF detector signal (million counts/s)

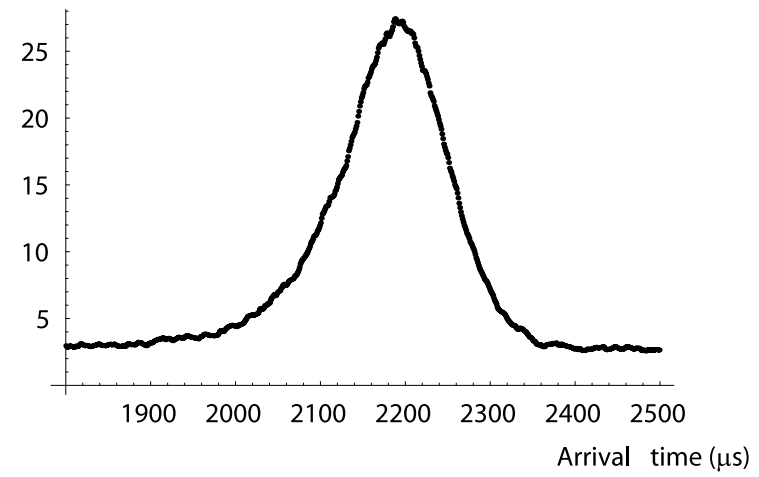

FIG. 2: A packet of molecules passing through the detector. Each molecule emits on average 1.9 photons before being lost to a dark state. Approximately $2 \%$ of the emitted photons are registered.

The interaction region, shown in Fig. 1, starts $450 \mathrm{~mm}$ from the skimmer and is $790 \mathrm{~mm}$ long. It is magnetically shielded to reduce the ambient field, and current-carrying wires inside the shields allow us to apply a magnetic field. Within this region there is a pair of electric field plates, $750 \mathrm{~mm}$ long and $70 \mathrm{~mm}$ wide, with a $12 \mathrm{~mm}$ spacing accurate to better than $200 \mu \mathrm{m}$ over the full length. These plates are machined from cast aluminium, then gold coated to improve the uniformity of the surface potential. The whole assembly is non-magnetic. With a field across the gap of $15 \mathrm{kV} / \mathrm{cm}$ the leakage current is less than $1 \mathrm{nA}$.

The same plate structure also serves as a $34 \Omega$ transmission line for the $170 \mathrm{MHz}$ radiation that drives the ground-state hyperfine transition. This transports the radiofrequency field as a fundamental TEM wave travelling parallel or antiparallel to the beam direction. Figure 3 shows how the rf is coupled in and out of the transmission line using high-voltage porcelain $100 \mathrm{pF}$ capacitors to connect to the $50 \Omega$ non-magnetic semi-rigid cable. The connections are shimmed with small $(\sim 10 \mathrm{pF})$ ceramic disk capacitors to minimise the reflections at each end. As a packet of molecules travels along the beamline, we can drive a hyperfine state changing transition at any desired position within the plate structure by pulsing the rf field on for a short period of time.

\section{TIME OF FLIGHT AND SPATIAL RESOLUTION}

It is a key element of our mapping technique that the pulses of molecules have small spatial and temporal ex-

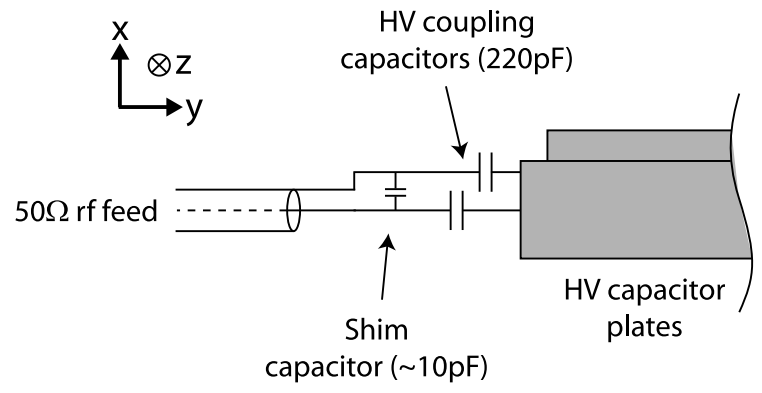

FIG. 3: rf transmission line coupling. Both ends of the capacitor plates are coupled to $50 \Omega$ co-axial feeds in this manner. $\mathrm{rf}$ is injected in one end of the capacitor and coupled out of the other. The direction can be reversed to check for systematic effects.

tent at the source. A very similar source used to make $\mathrm{LiH}$ produces the molecules in the $5 \mu$ s following the firing of the ablation laser. This was determined from studies with dual ablation pulses having a variable time separation [15]. We believe that the YbF molecules are formed within a similar temporal window. The initial size of the YbF pulse is measured by comparing the temporal profile of the molecular packet at two distances from the source, using the pump beam as one LIF detector and the probe as the other. Extrapolation back to the source then gives the initial spatial extent as $11 \mathrm{~mm}$ for an initial $5 \mu \mathrm{s} \mathrm{du}-$ ration and, in any case, not more than $12 \mathrm{~mm}$.

In demonstrating our field mapping method, we have typically used rf pulses of $18 \mu$ s duration. During this short time the molecules move only $11 \mathrm{~mm}$, allowing the rf transition to provide almost a freeze-frame picture of the molecular pulse at a particular position inside the interaction region. By studying the centre-frequency, lineshape and power dependence of the hyperfine transition we can infer the electric, magnetic and rf fields at that position. Repeating the experiment for a range of positions allows us to build a map of the field in the interaction region [18].

By binning the molecules according to arrival time, we can obtain maps with even finer spatial resolution. In the limit where all of the molecules are created at the same time and in the same place, the arrival time, $t_{\text {arr }}$, is simply a measure of the molecule's velocity. From this we can infer its position $z_{r f}^{\prime}$ at the time $t_{r f}$ of the rf pulse:

$$
z_{r f}^{\prime}=\frac{t_{r f}}{t_{a r r}} l_{m}
$$

where $l_{m}$ is the length of the machine from source to detector. The spatial resolution is limited by three factors: the detector has an active volume of non-negligible size and hence limited time resolution, the rf pulses are of finite length, and the beam source is not point-like in space or time. The uncertainty from the initial extent of the molecular pulse can be estimated as follows. Assume a molecule is formed at time $\Delta t$ and position $\Delta l$ with 
velocity $v$. The time of arrival at the detector will be

$$
t_{\text {arr }}=\frac{l_{m}-\Delta l}{v}+\Delta t .
$$

It is straightforward to show that our estimate of position at time $t_{r f}$ will differ from the molecule's true position, $z_{r f}$, by

$$
\Delta z_{r f}=z_{r f}^{\prime}-z_{r f}=\frac{t_{a r r}-t_{r f}}{t_{a r r}} \frac{l_{m} \Delta t-t_{a r r} \Delta l}{t_{a r r}-\Delta t} .
$$

Assuming $\Delta t$ and $\Delta l$ to be uncorrelated, with standard deviations $\sigma_{t}$ and $\sigma_{l}$ respectively and with zero mean, the uncertainty in the estimate of the position is given by

$$
\sigma_{z_{r f}}=\frac{t_{a r r}-t_{r f}}{t_{a r r}^{2}} \sqrt{t_{a r r}^{2} \sigma_{l}^{2}+l_{m}^{2} \sigma_{t}^{2}} .
$$

For our source, Eq. 1 yields an uncertainty of $11 \mathrm{~mm}$, which is equal to that from the rf pulse duration, giving a net positional uncertainty of $15 \mathrm{~mm}$ (the detector resolution is small). We note that the effect of finite detector size and rf pulse duration could be removed by an appropriate deconvolution, but that approach is not pursued here.

\section{FIELD MAPPING}

\section{A. Electric field measurement}

After the $F=1$ state population has been depleted by passing through the probe laser, it is replenished by the application of an rf pulse, which transfers population from the $F=0$ state. This causes an increase in the probe fluorescence, as shown in the rf frequency scan of Fig. 4. When considering the state of the molecule, it is appropriate to chose the static electric field direction as the axis of quantization because the electric dipole interaction is by far the strongest coupling to the external fields. Being a TEM wave, the rf magnetic field between

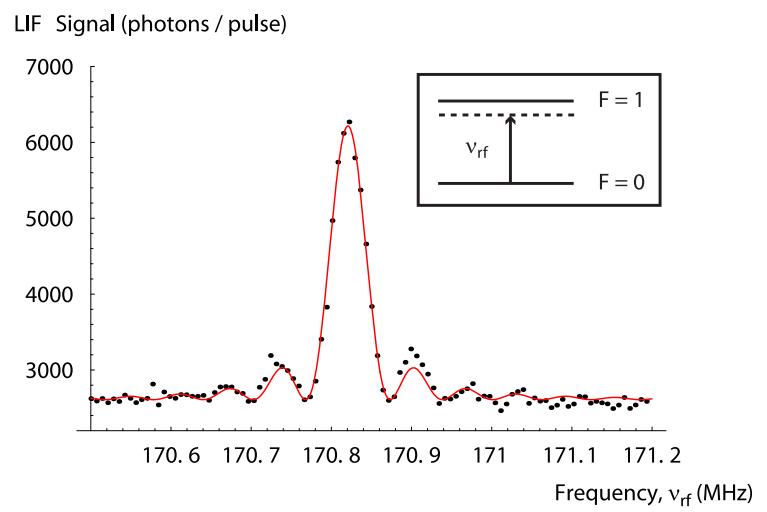

FIG. 4: Spectrum of the ground-state hyperfine transition. the plates is accurately perpendicular to the static electric field. As a result of this polarization, the rf field drives the transitions $(F=0) \rightarrow\left(F=1, m_{F}= \pm 1\right)$. Transitions to $\left(F=1, m_{F}=0\right)$ are strongly suppressed because of the polarisation and are in any case Stark shifted to a different resonant frequency. For the resonance shown in Fig. 4, the rf pulse was applied when the molecules were near the middle of the interaction region and the pulse amplitude was chosen to give the molecules a $\pi$-flip on the Bloch sphere at resonance. The transition probability peaks at $170.821 \mathrm{MHz}$, not at the field-free frequency of $170.254 \mathrm{MHz}$. Because the Stark effect of the $\mathrm{YbF}$ hyperfine levels is accurately known [16], this shift allows us to determine the strength of the electric field at the place where the molecules are. The Doppler shift is only a few hundred Hertz and is therefore insignificant at this level of accuracy. The curve shown in Fig. 4 is the lineshape calculated for a square rf pulse. This agrees quite well with the data points, though not perfectly. The deviations, mainly in the wings, are caused by the departure from a perfectly square rf pulse profile due to the few-microsecond settling time of the rf switches.

In figure 4, the LIF signal is the total photons counted per beam pulse, i.e. integrated over arrival time. Figure $5(\mathrm{a})$ shows the same data, but here the LIF signal is plotted versus arrival time as well as frequency [19]. It can be seen that the lineshape and line-centre are essentially independent of arrival time, indicating that molecules at the front of the packet (early arrivals) experience the same static electric field during the rf pulse as those at the back (late arrivals). This shows that the electric field is homogeneous in the centre of the interaction region. In Fig. 5(b) the rf pulse is applied just as the molecule leaves the high-voltage plates and the frequency scan is zoomed in on the central fringe of the resonance. Here we see that the centre frequency (indicated by the dots) is constant for the slow molecules, which are still between

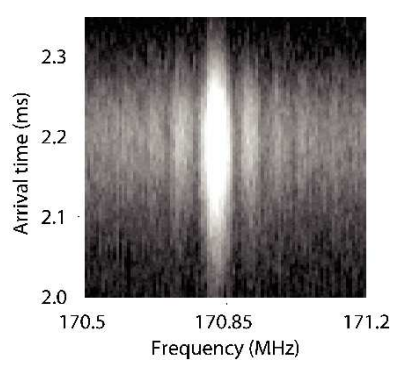

(a) Centre of machine

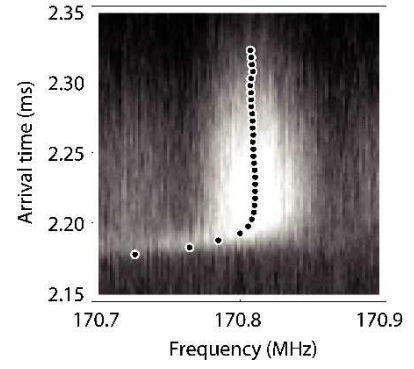

(b) Plate end
FIG. 5: (a) Spectrum of the ground state hyperfine transition as a function of frequency and arrival time. Integrating this data over arrival time yields Fig. 4 Integrating along the frequency axis gives a time-of-flight profile, similar to that of Fig. 2 (b) Hyperfine transition near the end of the interaction region. Note that both axes are expanded and shifted compared to those in (a). Circles show the fitted transition frequencies for several time slices. 


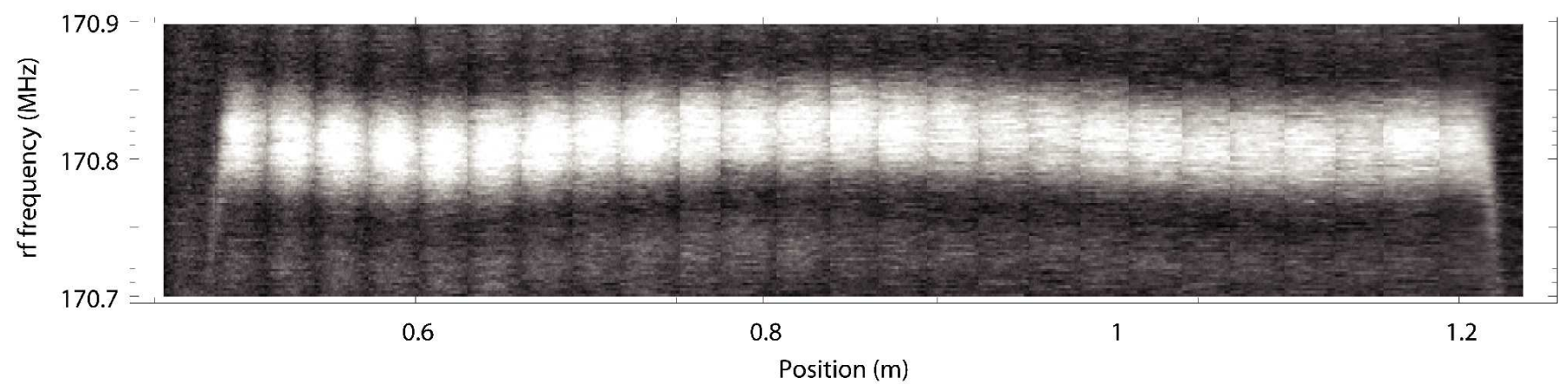

FIG. 6: Hyperfine transition spectrum as a function of position along the length of the interaction region. Joins between overlapping scans can be seen as dark vertical stripes.

the plates when the rf is pulsed on, but shifts dramatically for the fast molecules which explore the region of decreasing field at the exit of the plates.

This method of binning the data by arrival time allows us to map out a region, typically $6 \mathrm{~cm}$ in length, with a single frequency scan. In order to map the electric field over the whole interaction region, we repeat the scan for several rf pulse delays to make a sequence of short maps that can be stitched together, as shown in Fig. 6] This data set was collected in approximately 15 minutes. After fitting the line centre at each position and converting this to electric field [16], we obtain the field map plotted in Fig. 7. This shows very clearly a systematic $\pm 110 \mu \mathrm{m}$ variation of the plate spacing, which we can correlate with the design of the support mechanism. The importance of this measurement is that it shows the variation of electric field directly and in situ, which mechanical measurements on the bench cannot do.

The hyperfine transition frequency is sensitive to the electric field up to very high strengths: for $\mathrm{YbF}$ the gradient is $100 \mathrm{~Hz}$ per $\mathrm{V} / \mathrm{cm}$ at $30 \mathrm{kV} / \mathrm{cm}$. Because of this, polar molecules such as $\mathrm{YbF}$ offer unique sensitivity for probing very high fields. Uncertainty in the

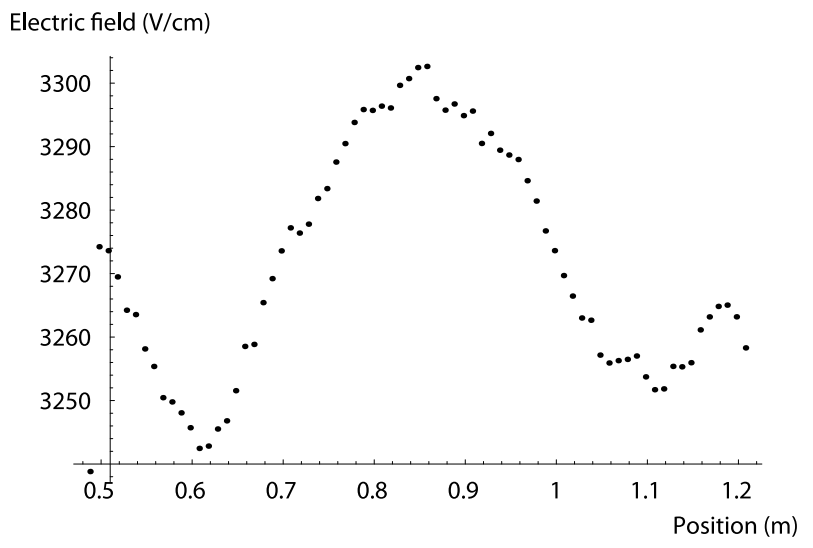

FIG. 7: Electric field as a function of position. The applied potential was roughly $\pm 2 \mathrm{kV}$ over the $12 \mathrm{~mm}$ gap. electric dipole moment of the molecule limits the absolute accuracy to approximately $1 \%$ in the case of $\mathrm{YbF}$ [16]. However, small relative field measurements are typically limited only by the accuracy of determining changes in the centre of the line. This in turn depends on the linewidth associated with the duration of the rf pulse. In the present experiment, using scans with $15 \mathrm{~mm}$ spatial resolution (18 $\mu$ s pulse), we achieve an uncertainty of $500 \mathrm{~Hz}$ with 30 s of measurement, corresponding to a relative field uncertainty of only $1.5 \mathrm{~V} / \mathrm{cm}$ at $3.3 \mathrm{kV} / \mathrm{cm}$.

\section{B. Static magnetic field}

If there is a magnetic field component $B_{z}$ parallel to the electric field, the $\left(F=1, m_{F}= \pm 1\right)$ energy levels are split by the Zeeman interaction. Although this does not shift the centre of the resonance, it does cause a splitting of the line, which can be used to measure $B_{z}$. By contrast, a perpendicular component of magnetic field has no significant effect on the lineshape and produces only a very small shift of the centre. This insensitivity to the perpendicular field is due to the strong tensor Stark effect, which shifts the $\left(F=1, m_{F}=0\right)$ state far away in energy compared with the off-diagonal magnetic coupling 11]. By switching off the electric field it is also possible to measure $B_{y}$, but we do not demonstrate that here.

In order to demonstrate magnetic field mapping, we apply a small static magnetic field, roughly parallel to the strong electric field, by passing a current $I$ through through copper wires that run parallel to the beam inside the innermost magnetic shield. In addition there is a residual ambient field from imperfect shielding, and perhaps from ferromagnetic impurities within the interaction region.

Figure 8 shows the resonance line measured near the centre of the machine with a Zeeman splitting of $25 \mathrm{kHz}$ due to the application of a $0.9 \mu \mathrm{T}$ field. The rf pulse duration was lengthened to $50 \mu$ s so that this splitting could be clearly resolved. The theoretical line profile describes the central part of the line quite adequately, but does not 


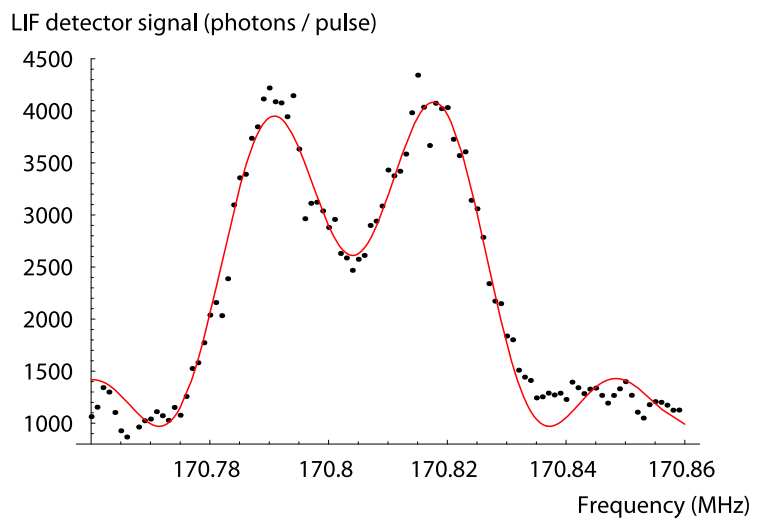

FIG. 8: Zeeman split hyperfine transition. The fit is to a sum of two square-pulse transition lineshapes.

fit well in the wings because it is once again based on a square rf pulse. In order to separate the ambient field from the applied field, we vary the current $I$ and fit the field to the form $B_{z}=\beta I+B_{0}$. Once this is completed at a given position, the rf pulse timing can be changed and the measurements repeated to build up maps of $\beta$ and $B_{0}$ along the beamline.

Figure 9 shows the results obtained after approximately 4 hours of data collection. The Zeeman splitting is fitted typically to within $100 \mathrm{~Hz}$. This fitting error leads to an uncertainty in $B_{0}$ of about $5 \mathrm{nT}$, and an uncertainty in $\beta$ of $100 \mathrm{pT} / \mathrm{mA}$. In addition, the absolute calibration of the field is limited by a $2 \%$ uncertainty in the g-factor. In Fig. 9(a), $\beta$ decreases at each end of the interaction region because the field rotates away from the z-axis to satisfy the boundary condition on the end-caps of the magnetic shield. The background field shown in Fig. 9(b) exhibits a sharp increase in magnitude at both ends of the interaction region. This was not expected and requires further investigation, though we surmise that it is due to inadequate demagnetisation of the magnetic shield end caps.

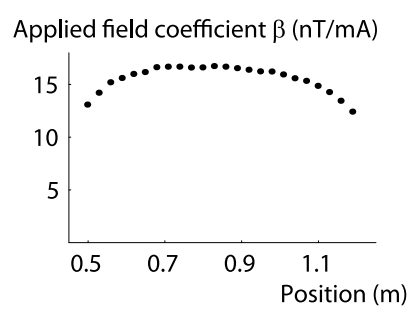

(a) Applied field

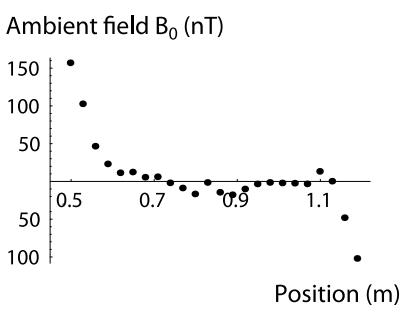

(b) Ambient field
FIG. 9: Maps of (a) applied field coefficient $\beta$ and (b) ambient field $B_{0}$ in the interaction region.

\section{C. rf magnetic field}

In the previous two sections, the centre and lineshape of the rf transition have informed us about the static electric and magnetic fields. In this section we use the dynamical evolution of the transition to provide information about the rf magnetic field itself.

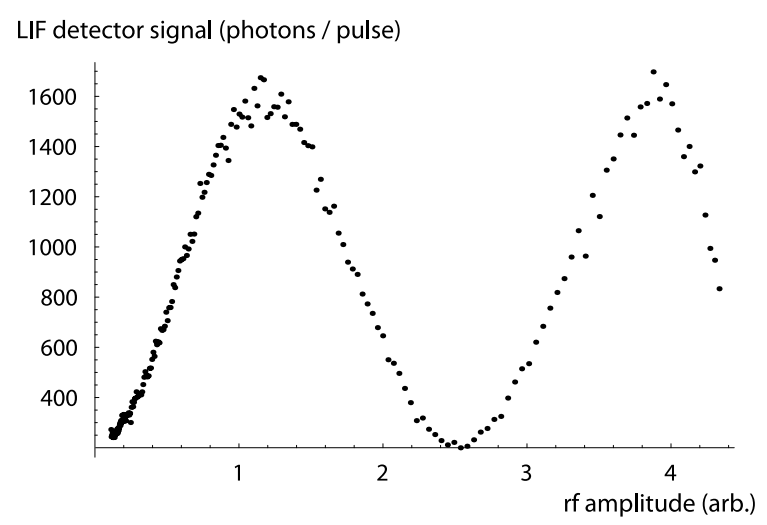

FIG. 10: Number of molecules in the $F=1$ state versus rf amplitude.

The rf field supported by the parallel plate transmission line could be a pure travelling wave in principle, but imperfect impedance matching at the ends inevitably produces some standing-wave component. It is therefore of interest to map the rf field strength as a function of position along the beamline. For this measurement, we fix the rf frequency at resonance and scan the amplitude to observe the Rabi flopping of the molecular population between the $F=0$ and $F=1$ hyperfine states. Figure 10 shows such a scan covering almost $4 \pi$ of Rabi flopping, with the rf pulse timed to excite molecules at the centre of the interaction region. The very good visibility of these fringes indicates that the rf field is rather homogeneous over the beam. In Fig. 11(a) we explore the longitudinal direction by replotting the data versus arrival time as well as field amplitude, much as we did

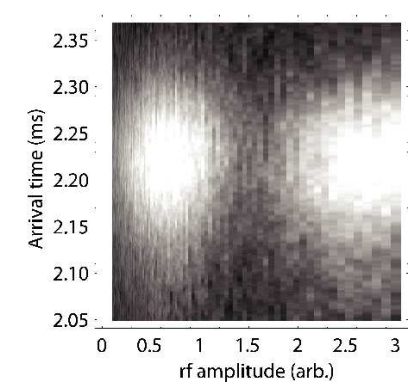

(a) Homogeneous field

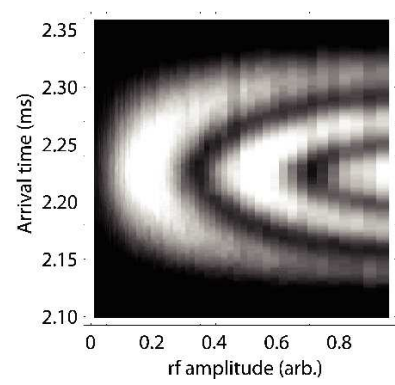

(b) Inhomogeneous field
FIG. 11: Rabi-flopping, resolved by arrival time: (a) driven by the homogeneous field of the transmission line, (b) driven by the inhomogeneous field of an $8 \mathrm{~cm}$ diameter loop. 


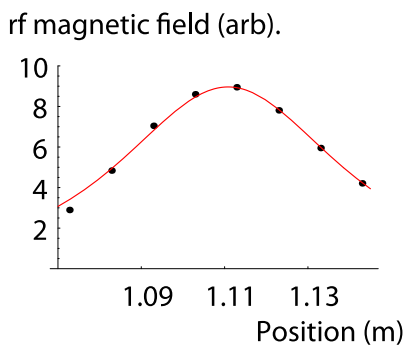

(a) Loop

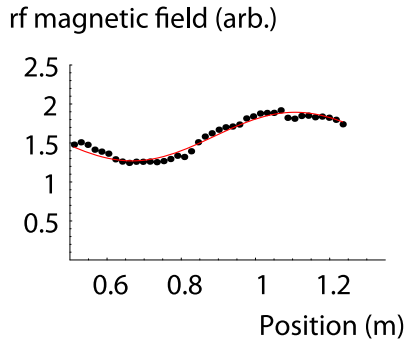

(b) Transmission line
FIG. 12: (a) Points: magnetic field of rf loop derived from data of fig. 11(b) Line: fit to dc field of a loop. Peak strength is the only parameter. (b) Points: rf field in the transmission line. Line: sine-curve corresponding to the field amplitude for a $170.820 \mathrm{MHz}$ standing wave. The amplitude and phase of the standing wave, and amplitude of the travelling wave were floated in the fit. Note the different $\mathrm{x}$-axis scale compared to (a).

in Fig. 5(a), This shows that molecules in different positions along the length of the packet have the same Rabi flopping rate, further demonstrating the uniformity of the field.

In contrast to this, Fig. 11(b) shows the result obtained with an inhomogeneous rf field generated by a copper loop encircling the field plates. The rf pulse timing was chosen so as to centre the molecules in the plane of the loop. One sees that molecules at the centre of the packet, with an arrival time of approximately $2.24 \mathrm{~ms}$, undergo a Rabi rotation of $5 \pi$ at the highest rf amplitude plotted, whereas molecules at the edges of the packet can barely make a single $\pi$-flip. The variation of the rf magnetic field amplitude reconstructed from this is shown in Fig. 12(a). The line superimposed on the data is the simple magnetic field variation expected for a DC field on the axis of a loop.

Returning to the transmission line, we have measured the rf field along the whole length of the interaction region by varying the timing of the pulse and making many overlapping scans. Figure 12(b) shows the result and clearly reveals the standing-wave component of the field. Superimposed on the data is a sine curve of half the freespace wavelength $(87.5 \mathrm{~cm})$, which fits well to the observed modulation. From this map, we estimate that the reflected amplitude at each end of the transmission line is $20 \%$, as one would expect in view of the impedance step between $50 \Omega$ and $34 \Omega$.

\section{CONCLUSION}

We have described a set of sophisticated diagnostic techniques that can be applied to pulsed atomic and molecular beams. These techniques have been developed in our lab to control and measure systematic effects associated with our electron-edm measurement. However, the main point of this article is to note the wider applicability of these methods to any experiment using pulsed atomic or molecular beams. In particular, these methods are very powerful in the context of high-precision atomic and molecular physics experiments, where pulsed beams have not hitherto found widespread application.
[1] G. Scoles (ed), Atomic and Molecular Beam Methods (Oxford University Press, 1988).

[2] N. F. Ramsey, Molecular beams (Oxford University Press, 1956).

[3] R. Wynands and S. Weyers, Metrologia 42, S64-S79 (2005).

[4] J. Vanier and C. Audoin, The Quantum Physics of Atomic Frequency Standards (IOP Publishing, 1989).

[5] C. S. Wood, S. C. Bennett, D. Cho, B. P. Masterson, J. L. Roberts, C. E. Tanner, and C. E. Wieman, Science 275, 1759 (1997).

[6] M. Niering et al., Phy. Rev. Lett. 84, 5496 (2000).

[7] M. Fischer et al., Phys. Rev. Lett. 92, 230802 (2004).

[8] Eric R. Hudson, H. J. Lewandowski, Brian C. Sawyer, and Jun Ye, Phys. Rev. Lett. 96, 143004 (2006).

[9] J. van Veldhoven, J. Küpper1, H.L. Bethlem, B. Sartakov, A.J.A. van Roij, and G. Meijer, Eur. Phys. J. D 31, 337 (2004).

[10] B. C. Regan, Eugene D. Commins, Christian J. Schmidt, and David DeMille, Phys. Rev. Lett. 88, 071805 (2002).

[11] J. J. Hudson, B. E. Sauer, M. R. Tarbutt and E. A. Hinds, Phys. Rev. Lett. 89, 023003 (2002).
[12] Norval Fortson, Patrick Sandars and Stephen Barr, Phys. Today. 56, 33 (2003).

[13] B. E. Sauer, H. T. Ashworth, J. J. Hudson, M. R. Tarbutt, and E. A. Hinds, in Atomic Physics 20, edited by Christian Roos, Hartmut Haeffner, Rainer Blatt (AIP, New York, 2006), AIP Conf. Proc. 869, p. 44.

[14] M. R. Tarbutt, J. J. Hudson, B. E. Sauer, E. A. Hinds, V. A. Ryzhov, V. L. Ryabov, and V. F. Ezhov, J. Phys. B 35, 5013 (2002).

[15] S. K. Tokunaga, J. O. Stack, J. J. Hudson, B. E. Sauer, E. A. Hinds, and M. R. Tarbutt, J. Chem. Phys. 126, 124314 (2007).

[16] B. E. Sauer, J. Wang and E. A. Hinds, J. Chem. Phys. 105, 17 (1996).

[17] S. R. Jefferts et al., Metrologia 39, 321 (2002).

[18] We note that a somewhat related technique has been used to map the magnetic field in an atomic fountain clock [17], but this was limited to the region of the atomic cloud's apogee.

[19] These and all subsequent density plots have been gammatransformed and windowed to improve the contrast. 